\title{
The Complex Nature of Breast Cancer Stem-Like Cells: Heterogeneity and Plasticity
}

\section{Rhiannon French and Richard Clarkson*}

School of Biosciences, Cardiff University, Wales, UK

\begin{abstract}
There are multiple definitions of cancer stem cells based on the different assays used to detect or enrich for them. The existence of different assays has made the identification and isolation of the archetypal cancer stem cell (CSC) with all of these properties an attractive but as yet unachievable goal. Indeed, it has been suggested that the lack of complementarity between these assays is in itself a barrier to CSC identification. Yet new insights into the heterogeneity of breast cancer stem cells and the discovery of CSC plasticity now suggests that rather than the existence of a single elusive stem-like entity in cancers, there may be a heterogeneous mix of cell populations able to switch their phenotype under different selective pressures. The aim of this review is to summarise the current evidence supporting this hypothesis and to suggest that focusing on the mechanisms controlling the inter-conversion between these minority stem cell populations could lead to more effective strategies to target the malignant properties of breast cancer stem cells.
\end{abstract}

Keywords: Cancer stem cell; Breast cancer; Heterogeneity

Abbreviations: CSC: Cancer Stem Cell; bCSC: Breast Cancer Stem Cell; miRNA: microRNA; ESA/EpCAM: Epithelial Specific Antigen; HMECs: Human Mammary Epithelial Cells; EMT: Epithelial to Mesenchymal Transition

\section{Introduction}

\section{Models of tumourigenesis and the cancer stem cell hypothesis}

The cancer stem cell (CSC) hypothesis first arose as a result of the noted similarities between normal stem cells and tumourigenic cells: both have the ability to self renew and to generate the heterogeneous cell populations observed in tissues and tumours. These similarities together with the rarity of stem cells in adult tissues are what led to the notion that there may be only a subset of cancer cells within a tumour with tumourigenic capacity [1]. This theory is in accordance with the cell-of-origin model of tumourigenesis which is one of two theories proposed to account for the generation of heterogeneity observed within tumours such as those of the breast (Figure 1) [2]. The cell-oforigin model states that intra-tumoural diversity derives from a single cell sitting at the top of a cellular hierarchy similar to that observed in the normal mammary epithelium. The tumour cell-of-origin is often considered synonymous with the CSC and although this may not necessarily continue to be the case throughout tumourigenesis, it is by definition the first CSC.

Alternatively, it has also been proposed that some cancers may arise in a non-hierarchal manner, whereby transforming genetic lesions occur to multiple cells during the process of tumourigenesis. This model of clonal evolution was initially put forward as an opponent of the cell-of-origin hypothesis; however, the two need not be mutually exclusive. It is possible that the phenotypic heterogeneity of a tumour may arise as a result of both the phenotype of a cell of origin (i.e. the CSC), and that conferred by evolution (Figure 1).

\section{Discovery of breast cancer cells with stem-like properties}

Experimental evidence in support of the cell-of-origin hypothesis came about in 1997 with the discovery of putative CSCs in acute myeloid leukaemia. Bonnet and Dick [3], by virtue of the cell surface markers $\mathrm{CD} 34^{+} / \mathrm{CD} 38$, isolated a population of cells with enhanced tumourigenic capacity following transplantation into NOD/SCID mice. Al Hajj et al. [4] subsequently recapitulated this observation for the first time in solid tumours by enriching for a sub-population of tumour initiating cells from primary breast lesions using the cell surface marker profile $\mathrm{CD} 44^{+} / \mathrm{CD} 24^{-} / \mathrm{ESA}^{-} /$lin- (negative for several lineage markers and therefore, considered undifferentiated). Tumourigenic sub-populations have since been discovered in a variety of cancer tissue types, including brain, colon and pancreas [5-8].

\section{Definition and identification of breast cancer stem-like cells}

A normal stem cell is defined by its ability to divide both

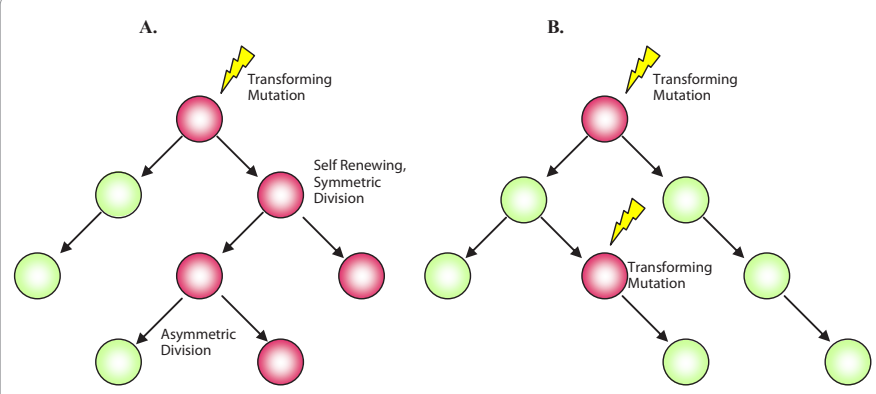

Figure 1: Models of tumorigenesis: A. The cell-of-origin hypothesis; transforming mutations occur to a single cell which acquires or deregulates stem-like characteristics allowing it to divide symmetrically to self-renew or asymmetrically to generate differentiated cells in order to propagate and form a tumour. B. The clonal expansion hypothesis; transforming mutations occur to a number of cells during the process of tumorigenesis.

*Corresponding author: Richard Clarkson, School of Biosciences, Cardiff University, Wales, UK, E-mail: ClarksonR@cf.ac.uk

Received December 01, 2012; Accepted January 11, 2013; Published January 13, 2013

Citation: French R, Clarkson R (2012) The Complex Nature of Breast Cancer Stem-Like Cells: Heterogeneity and Plasticity. J Stem Cell Res Ther S7:009. doi:10.4172/2157-7633.S7-009

Copyright: (C) 2012 French R, et al. This is an open-access article distributed under the terms of the Creative Commons Attribution License, which permits unrestricted use, distribution, and reproduction in any medium, provided the original author and source are credited. 
symmetrically to self-renew and asymmetrically to generate differentiated cells $[9,10]$. A cancer stem cell should therefore also be defined as such, possibly adding the property of extensive proliferation $[1,11]$. Indeed, CSCs are more prevalent in mammary tumours compared to their normal counterparts in mammary tissues, a property that is attributed to an increased rate of self-renewal of breast cancer stem cells (bCSCs) possibly linked to p53 activity [12].

The gold standard of CSC identification, including breast cancer stem cells (bCSCs), continues to be tumour initiation with serial transplantation in recipient mice and most of the literature relies upon tumour initiation in vivo to identify and quantify stem-like cells from tumour cell populations. This phenotypic trait encompasses the key properties of self-renewal, asymmetric division and enhanced proliferative capacity. However, as it is impossible to identify and subsequently isolate CSCs based purely on this premise, a number of methods of identification and enrichment have been adopted that rely on additional CSC-phenotypic properties associated with the ability to propagate new tumour growth in vivo (Table 1).

Despite this, the identification and isolation of cancer stem cells remains problematic. This has been attributed in part to bCSC heterogeneity both within and between breast tumours. In addition to heterogeneity, there is a mounting body of evidence to suggest that these cell populations are also dynamic; both stem, and non-stem-like populations may be capable of inter- or intra-conversion in response to environmental cues $[13,14]$.

In this review we will summarise the experimental evidence for heterogeneity and plasticity of breast CSCs and discuss the implications of these properties both for future characterisation of bCSCs and the development of effective therapeutic strategies targeting them (Table $1)$.

\section{Heterogeneity of breast cancer stem-like cells}

Despite the continued use of the term "cancer stem cell" to describe the minority of self-renewing, tumour-initiating, cells within a tumour, it is becoming apparent that the stem-like characteristics that accompany this description do not necessarily occur within the same sub-population of cells. This suggests that our ability to identify and then to isolate "cancer stem cells" is impeded by limitations in our current CSC assays and that the existence of stem-like characteristics within tumours is a more complex situation than initially thought. Here we summarise the evidence for marked differences in the number and phenotype of stem-like cells between breast tumours and the coexistence of cancer cells with distinct stem-like properties within breast tumours.

\section{Inter-tumour bCSC heterogeneity}

The identification of targetable stem-like features relies upon the ability to isolate and study stem-like cell populations. The identification of signature "stem-like" cell-surface protein expression profiles has made it possible to enrich for breast cancer cells with increased tumourigenic and self-renewal capacity. However, the heterogenous nature of breast cancer makes the existence of a universal stem cell surface marker profile unlikely. Breast cancer has been divided into different subtypes based on tumour cell type; basal-like, ErbB2+, normal breast like, luminal subtype $\mathrm{A}$ and luminal subtype $\mathrm{B}$, and the more recently identified claudin-low subtype $[15,16]$. To date a number of different marker profiles have been used to isolate stem-like cells from breast cancers, each with varying degrees of specificity across the molecular subtypes [17].

The first identification of bCSCs was achieved by the isolation of cells with the cell surface marker profile of $\mathrm{CD} 44^{+} / \mathrm{CD} 24^{-/ \mathrm{ESA}^{+} / \mathrm{Lin}}$ [4]. Subsequent studies have shown that this profile does not enrich for tumour initiating cells in all breast tumours, but instead is particular to basal-like breast cancer; for example, in one study the profile was only identified in $31 \%$ of 240 samples where it correlated with basal subtypes [18]. Honeth et al. [18] analysed the expression of CD44 and CD24 across a range of breast tumour samples by immunohistochemistry. The stem-like phenotype of $\mathrm{CD} 44^{+} / 24^{-}$correlated strongly with low Her2 expression and elevated EGFR and was predominantly found in the basal-like/Her2 negative subgroup as defined by gene expression analysis of tumour samples. The CD $44^{+} / 24^{-}$phenotype was underrepresented in Her2 positive tumours, which instead had a strong association with CD $44^{-/ 24^{+}}$expression. In addition, $94 \%$ of BRCA null and all medullary tumours expressed the CD44 ${ }^{+} / 24$ phenotype [18].

In contrast to the findings of $\mathrm{Al} \mathrm{Hajj} \mathrm{et} \mathrm{al.} \mathrm{[4],} \mathrm{Meyer} \mathrm{et} \mathrm{al.}$ demonstrated in an ER-negative breast tumour sample that in the CD $44^{+}$population, both CD24 negative and positive cells exhibited equivalent tumorigenic capacity and could be further enriched with markers for CD49 and CD133; markers associated with normal mammary stem cells $[19,20]$. Cariati et al. [21] also demonstrated a role for CD49f as a marker of breast cancer stem cells in the (ERa positive) MCF-7 cell line, whereby mammospheres exhibited a greater

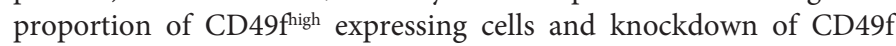
completely prevented mammosphere formation and tumour initiation in vivo [21].

Similarly, CD133 (prominin-1), a marker of CSCs in haematological malignancies and a variety of solid tumour types [22], was associated with bCSCs derived from tumours in a Brcal deficient mouse model [23]. Cell lines derived from these tumours exhibited a CD133 positive sub-population highly enriched for tumour initiating cells. CD133 positive cells were shown to have stem-like properties comparable to the $\mathrm{CD} 44^{+} / \mathrm{CD} 24$ populations isolated from other Brca null tumour cell lines, including similar gene expression profiles, although significantly no overlap between the $\mathrm{CD} 44^{+} / 24^{-}$and $\mathrm{CD} 133^{+}$population was

\section{Proposed Stem-like Characteristics}

Tumour initiation/ maintenance of tumour growth

\section{Corresponding Assays}

- In vivo transplantation

The capacity for both symmetrical division (self renewal) and assymmetrical division be assayed for: (differentiation)

- in vitro by the tumour sphere assay [48]

- in vivo by serial transplantation

\section{Resistance to Anoikis}

(the form of apoptosis induced by cellular detachment)

Metastasis formation

(Combination of anoikis resistance and tumour initiation)

- In vitro Tumour sphere assay [48]

- In vivo analysis of circulating tumour cells

Drug resistance

- in vivo metastasis models e.g. by tail vein or intra-cardiac injection

- In vitro/in vivo Cell survival following drug treatment

Table 1: Stem-like characterisitics associated with tumour cells and the corresponding assays used to test for them. 
observed despite all tumours occurring in BRCA null mice. Similarities in gene expression included over-expression of seven stem-cell related genes such as Notch1, Fgfr1, CD44, Sox1 and Aldh1a1, although there were some notable differences including 17 -fold higher levels of KRT5 in CD133 positive subsets compared to CD44/CD24 cells [23].

Aldehyde dehydrogenase (ALDH) was identified as a marker of tumorigenicity by Ginestier et al. [24]. ALDH is an enzyme which detoxifies aldehydes such as retinol, and was studied because of its previous associations with leukaemic stem cells [25]. In this study, it was found that $3-10 \%$ of the cell population of each of four primary tumour samples ( 3 triple negative and 1 ERBB negative) was ALDH positive. When isolated, ALDH positive cells were found to exhibit at least 100 -fold greater tumour initiating capacity than their ALDHnegative counterparts. Although limited growth was observed with 50000 ALDH negative cells, resulting tumours did not self renew suggesting that tumour initiation may have been instigated by a progenitor population [24]. ALDH positive populations have since been identified in 23/33 cell lines where as consistent with the CD $44^{+} /$ $\mathrm{CD} 24 / \mathrm{ESA}^{+} / \mathrm{Lin}^{-}$profile it correlated with those lines of a basal-like nature, e.g. as few as 100 ALDH positive cells of the basal-like SUM159 cell line were required to generate tumours. Although ALDH is associated with elevated ERBB expression in breast cancers this was not found to be the case in cell lines [26].

The aforementioned studies all refer to isolation of bCSCs from basal-like and more aggressive disease subsets and despite some sporadic success, the current marker profiles do not identify stem-like cell populations from luminal-like breast cancers [18,27]. It is possible that this may allude to the existence of an alternative mechanism of tumorigenesis in luminal tumours, such as clonal evolution (Figure 1). This theory is supported by the MMTV-Erbb2 mouse model of breast cancer which spontaneously generates relatively homogenous luminal tumours from which no distinct rare subpopulation of stem-like cells can be isolated; in fact, these tumour cells are tumorigenic with as few as 100 cells [28]. However, it has been suggested that as relapse is common in patients with luminal-like breast cancers, the stem-like component of this subset exists but still remains elusive [29].

The range of marker profiles currently used to enrich for stem like characteristics across tumours could be an indication that we have not yet identified the universal bCSC marker profile. However, it is more likely that the bCSC population mirrors that which is observed among all breast cancer cells and is heterogeneous in nature. Further studies of bCSC marker profiles, perhaps those incorporating gene expression analyses, will undoubtedly increase our understanding of heterogeneity and may even make it possible to classify breast tumours based on distribution of bCSC characteristics (Table 2).

\section{Intra-tumour heterogeneity in cancer stem-like cells}

The cell-of-origin model of tumorigenesis assumes that CSCs are a rare population at the top of a cellular hierarchy, but this concept is now being challenged. In addition to the bCSC heterogeneity observed between breast tumours, there is some evidence to suggest that there exists more than one population of stem-like cells even within a single tumour or cell line. Intra-tumour CSC populations have come to light by investigating different aspects of stem-like behaviour within a single tumour which by our current definitions do not completely overlap. Intra-tumour heterogeneity is most easily observed by cellsurface marker profile analysis due to the ability to analyse both potential populations simultaneously in the same environment. With a sequential analysis it is not possible to determine whether two stemlike populations exist or whether plasticity has occurred; i.e. in the time it takes to perform transplantation assays, a stem-like cell could have derived from a non-stem.

Although intra-tumour diversity in stem like cells has been observed in other cancers [30], evidence for intra-tumour CSC heterogeneity in breast cancer has only arisen from a few papers including the aforementioned study of cell surface markers by Ginestier et al. [24]. In the four samples investigated, the ALDH positive population only overlapped with the $\mathrm{CD} 44^{+} / \mathrm{CD} 24 / \mathrm{ESA}^{+} / \mathrm{Lin}^{-}$profile by $0.1-1.2 \%$. The ALDH negative population was not tumorigenic whereas the ALDH + required 500 cells to form tumours, but the overlapping population required only 20 cells to produce tumours [24]. On the basis of these findings, two interconverting stem-like subpopulations have been proposed, each possessing distinct stem-like features (Figure 2). In a recent review, Liu et al. [31] have related bCSC markers to normal mammary stem cell markers and found that the bCSC population as defined by $\mathrm{Al} \mathrm{Haij} \mathrm{et} \mathrm{al.} \mathrm{[4]} \mathrm{resides} \mathrm{within} \mathrm{the} \mathrm{EpCAM-CD49f+}$ population, and ALDH positive within the EpCAM+CD49f-population, the latter being considered more epithelial-like. On this basis bCSCs have been divided into more luminal or MET-like and more basal or EMT-like. It is interesting to note that despite the inability to initiate tumour growth, the $\mathrm{ALDH} / \mathrm{CD} 44^{+} / \mathrm{CD} 24 / \mathrm{ESA}^{+} / \mathrm{Lin}^{-}$population is still considered stem-like by this group. Furthermore, there is evidence to suggest that these populations reside at different areas or niches of the tumour: the MET-like at the centre, and the EMT like at the invasive front [31]. The possible dynamics of this system are discussed in more

\begin{tabular}{|c|c|c|c|}
\hline Marker & Target & $\begin{array}{l}\text { Cell numbers required for tumour formation } \\
\text { in vivo }\end{array}$ & Reference \\
\hline \multirow[t]{2}{*}{ CD44+/CD24-/ESA+ } & $\begin{array}{l}\text { Human breast cancers: } \\
\text { Basal-like, claudin low, Her2-ve, BRCA null, and medullary tumours }\end{array}$ & 200 & {$[4,18]$} \\
\hline & Cell lines & Not tested & [17] \\
\hline $\mathrm{CD} 44^{+} / \mathrm{CD} 49 \mathrm{f}^{\mathrm{fi}} / \mathrm{CD} 133^{+}$ & $E R a-$ tumours & 250 & [19] \\
\hline $\mathrm{CD}_{49 \mathrm{f}^{+}}$ & MCF-7 cell line & Not tested & [21] \\
\hline CD133+ & BRCA- mouse tumours & 50 & [23] \\
\hline \multirow[t]{2}{*}{$\mathrm{ALDH}+$} & $\begin{array}{l}\text { Human breast cancers: } \\
\text { Triple negative } \\
\text { ERBB2 negative }\end{array}$ & 500 & [24] \\
\hline & Cell lines e.g. SUM159 & 100 & [26] \\
\hline $\begin{array}{l}\text { CD44+/CD24-/ESA+ } \\
\text { ALDH }+\end{array}$ & Human breast cancers & 20 & [24] \\
\hline PROCR+/ESA+ & MDA-MB-231 cell line & 100 & [17] \\
\hline
\end{tabular}

Table 2: Markers used to isolate breast cancer cell subpopulations enriched for tumorigenic capacity. 


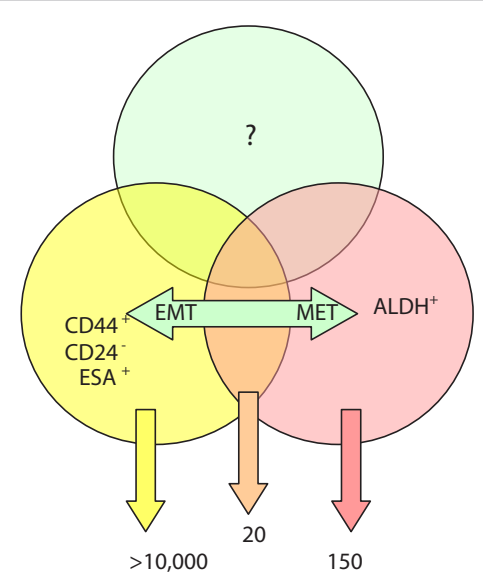

No. cells required to

fo rm tumours in vivo

Figure 2: Intra-tumour heterogeneity and plasticity of stem-like populations: Two or more interconverting stem-like populations may exist within breast tumours [24,31]. Further investigation may reveal yet more stem-like subpopulations but a universal CSC marker may be an unrealistic prospect.

\section{detail in subsequent sections.}

Further evidence which may support this theory comes from a study of rat mammary tumours by Zucchi et al. [32]. Three clonal subpopulations were isolated from a rat mammary tumour derived from rat LA7 bCSCs; stem-like, mesenchyme-like and epithelial like. Both the stem-like and epithelial-like cells although morphologically distinct were able to initiate tumour growth in vivo and to form tumorspheres, however the epithelial-like cells were incapable of self-renewal in both assays. This is another example of a cell population exhibiting some but not all stem-like attributes. The lack of self-renewal capacity in this population led the authors to describe it as a progenitor-like but nonetheless tumorigenic population. In addition, the epithelial-like population expressed a cell-surface expression signature comparable to that observed in the core of the tumour, whereas the stem-like population was comparable to the tumour invasive front, thus echoing the findings of Liu et al. [31] as mentioned above [32].

\section{Plasticity of Breast Cancer Stem-like Cells}

\section{Plasticity between non-stem-like breast cancer cells and bCSCs}

A certain degree of plasticity may exist within a tumour cell population which allows interconversion between CSC and non-CSC states when driven by selective pressures (including therapy) or clonal evolution. Indeed CSCs within a tumour may be a different entity at instigation of tumorigenesis compared to diagnosis or following therapy [11]. The study of intra-tumour plasticity is more difficult than tumour origin as it could easily be confused with a potential heterogeneity of stem-like populations and therefore cannot simply be observed by an alteration of stem-like characteristics in the total population. To study this process, subpopulations of cancer cells must be assayed separately. If a certain stem-like characteristic were to appear in a population previously devoid of such attributes, only then would it be possible to conclude that plasticity has occurred.

An analysis of the plastic potential of HMECs was carried out in this manner by Chaffer et al. [13]. Initially, non-transformed HMECs were considered and the cell population first sub-divided by the isolation of a small population of cells found floating in the media of HMEC cultures. The floating cells (designated HME-Flopc) were found to contain a greater proportion of stem-like cells as determined by the expression of the stem cell markers CD $44^{+} / \mathrm{CD} 24 / \mathrm{ESA}^{+},(2.5 \%$ compared to $0.2 \%$ ). This correlated with approximately 1.5 in 300 cells having mammosphere forming potential compared to none in the remaining population. Clonal flopc populations were subsequently fractionated into $\mathrm{CD} 44^{\mathrm{lo}}$ and $\mathrm{CD} 44^{\mathrm{hi}}$ subsets. Re-assaying for marker profile following adherent culture revealed CD $44^{\text {hi }}$ cells in the CD $44^{\text {lo }}$ fraction. In addition, even in the presence of a high proportion of labelled CD44 $4^{\text {hi }}$ cells, CD $44^{\text {hi }}$ cells could still be generated from unlabelled $\mathrm{CD} 44^{\text {lo }}$ cells. To analyse the oncogenic counterparts of flopc cells, oncogenic transformation was induced by introduction of the SV40ER and H-Ras oncogenes. Transformation resulted in 5 -fold more efficient conversion of CD $44^{\text {lo }}$ cells to $\mathrm{CD} 44^{\text {hi }}$. This was also found to be true in vivo; CD44 $4^{\text {lo }}$ cells injected into mice formed tumours with up to $16 \% \mathrm{CD} 44^{\mathrm{hi}}$ cells [13]. This study was the first to definitively demonstrate plasticity upwards in the established hierarchy of both normal and neoplastic cell populations. These findings have important implications for breast cancer treatment as they suggest that targeting only the stem-like population of cancer might not be enough to eradicate a tumour of its metastatic potential, as stem-like cells could then re-generate from the non-stem population.

These observations have been corroborated by a similar analysis of the breast cancer cell lines SUM149 and SUM159 [14]. Both lines were fractionated into subpopulations based on the marker profile identified by $\mathrm{Al}$ Hajj et al. [4]: luminal: $\mathrm{CD} 44 / \mathrm{CD} 24^{+} / \mathrm{ESA}^{\mathrm{H}}$, basal: $\mathrm{CD} 44^{+} /$ $\mathrm{CD} 24 / \mathrm{ESA}^{-}$, and stem-like; $\mathrm{CD} 44^{+} / \mathrm{CD} 24^{-} / \mathrm{ESA}^{+}$. These subtypes were confirmed by gene expression analysis; however no functional assay was used. All isolated populations were able to transition back to a heterogeneous population which recapitulated the proportions of each subset observed in unsorted cells. It was not likely that any stemlike component remaining within the basal or luminal populations increased via enhanced self-renewal due to no difference in proliferation being observed. As with the Chaffer study, these data indicate that it is possible for cells to transition between states, and most importantly have revealed that plasticity can occur between non-stem cancer cells and bCSCs. The Gupta et al. [14] study generated a mathematical model to describe state transitions and make predictions about luminal or basal cell transitioning to a stem-like state that were corroborated by in vivo experiments; luminal or basal subpopulations were able to form tumours when injected with non-tumorigenic irradiated GFP carrier cells used to improve survival [14]. This strongly indicates plasticity occurring within these populations however it cannot be ruled out that a tumorigenic subset remained within the purified populations, even without a stem-cell profile. The $\mathrm{CD} 44^{+} / \mathrm{CD} 24 / \mathrm{ESA}^{+}$phenotype at-best enriches for tumorigenic cells and tumours can arise from the stem-like negative population [4]. Furthermore, inhibition of TBX3 (a gene involved in the regulation of differentiation) by shRNA was able to perturb plasticity by decreasing the probability of luminal to basal transitions in SUM159 cells and increasing the probability of basal to luminal transitions in SUM149 cells. Although not able to prevent conversion to a stem-like state, this demonstrates a useful tool for the identification of genes involved in the process of plasticity as potential therapeutic targets. In addition this study was able to show that chemotherapy resulted in a 5-fold increase in the proportion of stem-like cells.

A number of studies have since indicated the importance of an EMT (epithelial-to-mesenchymal transition) like process in the 
conversion of cells to a CSC state. EMT is a process of cell transition which occurs primarily during embryogenesis, but can also take place during wound healing and tumorigenesis [33]. In tumorigenesis EMT has been implicated in metastasis of disseminated cancer cells, which are thought to require stem-like characteristics such as anoikis resistance. This correlation led to the notion that EMT may be responsible for imparting CSC traits. Mani et al. [34] investigated this possibility by induction of EMT in HMECS by overexpression of twist, a transcription factor known to be important in EMT. In addition to expected downregulation of epithelial markers and upregulation of mesenchymal markers such as $\mathrm{N}$-cadherin, cells acquired a stemlike phenotype and formed over 30 fold greater number of spheres than wild type cells. Induced EMT in Her2neu immortalised HMLEs resulted in a 10-fold increase in spheres and 2-fold increase in tumour initiating cells [34]. These data strongly implicate a role for EMT in plasticity however it cannot be distinguished from this study whether induction of EMT has increased self-renewal of existing bCSCs or plasticity towards a stem-like phenotype. The lack of difference between proliferation rates between subpopulation supports the latter possibility however an analysis of isolated subpopulations would be more conclusive. Morel et al. [35] supported this line of investigation by focussing on CD24 cells which were completely absent from HMECS. Transformation by the Ras oncogene resulted in the generation of a CD24 population which increased with time up to $65 \%$ of the population. This phenotype was consistent with stemlike cancer cells; CD24 cells could form mammospheres and tumours in vivo whereas $\mathrm{CD} 24^{+}$cells could not. Furthermore CD24- cells could be generated from $\mathrm{CD} 24^{+}$cells. Enrichment in CD24 cells following transformation coincided with morphological changes and alteration of gene expression profiles consistent with EMT. Experimentally induced EMT by TGF $\beta$ also resulted in the appearance of CD24 cells and an increase in the expression of mesenchymal markers. TGF $\beta$ also accelerated transformation by Ras [35]. Similar observations linking plasticity and EMT have been made in cell lines [36]. More recently, Yang et al. [37] have visualised plasticity between non-stem cancer cells and bCSCs occurring in situ in MCF-7 cells and shown that this process could be enhanced by the induction of EMT or perturbed by its inhibition [37].

The link between EMT and the formation of cancer stem cells has now been recognised but the molecular mechanisms underlying this plasticity have yet to be firmly established. To date only a few groups have addressed this issue. A number of molecular mechanisms which potentially underlie this stem cell/EMT plasticity have been identified including the $\mathrm{p} 130 \mathrm{Cas} /$ Cyclooxygenase- 2 axis and Brd4 $[38,39]$. Meyer et al. [19] have also demonstrated that single non-invasive epitheliallike $\mathrm{CD} 44^{+} / \mathrm{CD} 24^{+}$cells of breast cancer cell lines can generate invasive, mesenchymal-like CD $44^{+} / \mathrm{CD} 24$ cells in culture and following transplantation in vivo. Importantly, inhibition of activin/nodal signalling prevented the generation of heterogeneity from $\mathrm{CD}_{4} 4^{+} /$ $\mathrm{CD} 24^{+}$cells which could only then generate vimentin-low progeny. Inhibition was also able to prevent the generation of vimentinnegative progeny from $\mathrm{CD} 44^{+} / \mathrm{CD} 24^{-}$cells [19]. This study revealed the importance of activin/nodal signalling in plasticity which highlights an additional potential therapeutic target.

The most consistent evidence for regulation of stem cell/ EMT plasticity so far has come from investigations into the role of microRNAs in this process. MicroRNAs are untranslated RNAs which regulate the expression of multiple coding regions of the genome and have been implicated in the regulation of many cellular processes including stem cell division and also in carcinogenesis [40]. Chang et al. [41] identified two miRNAs, 200c and 183 as being the miRNAs most downregulated in the $\mathrm{CD} 44^{+} \mathrm{CD} 24$ population of HMECs and the normal mammary cell line MCF12A. It was subsequently established that the transcription factor $\mathrm{p} 53$ was able to bind promoter regions of both miRNAs. Overexpression of p53 was able to increase miR200c and 183 expression and reduce the expression of the stem cell-related genes, Klf4 and Bmil, both known targets of miR200c and 183. In addition, enforced EMT by TGF- $\beta$ treatment resulted in a downregulation of p53 and miR200c and 183, and elevated expression of Bmil and Klf4. shRNA inhibition of p53 was able to induce EMT and increased the percentage of stem cells and overexpression of miR$200 \mathrm{c}$ was able to rescue the phenotype of p53shRNA cells. Conversely, overexpression of p53 in TGF- $\beta$-treated cells was able to reverse the EMT process (i.e. MET) and reduced the percentage of stem cells. The relevance of this process in breast cancer was established by the observation that overexpression of mutant p53s found in breast tumours had the same affect as inhibition of wild-type p53. These data are evidence of the important relationship between p53 and miRNAs in the regulation of the conversion of non-stem breast cancer cells to bCSCs [41]. Polytarchou et al. [42] investigated the role of miRNAs in a cell line model of inducible bCSC formation: activation of the Src oncogene in the MCF10A line induces a cellular transformation which includes the production of bCSCs. By transfecting bCSCs from this model with a library of 355 miRNAs, a number were identified as capable of inhibiting bCSCs growth by at least $50 \%$, these included miRs 200, let7, 15, 16, 103, 1107, 145, 335 and 128b. Expression of these miRNAs were also reduced in bCSCs compared to non-stem cancer cells. In addition they shared a number of stem-cell associated target genes in common including Suz12, Zeb2, Klf4, and Bmil, and miRNA overexpression resulted in their downregulation. Low levels of these miRNAs and high levels of their targets were observed in bCSCs isolated from breast tumour samples but this inverse relationship was most significant in tumour samples which were triple-negative or basal-like. Although not direct evidence of plasticity, any plasticity occurring between the non-stem and bCSC populations investigated may require the downregulation of these microRNAs [42].

The aforementioned studies all used cell surface marker profiling to isolate stem-like populations and whilst this has proved an effective method to identify plasticity within tumours the evidence is mostly limited to a single stem-like attribute. Lineage tracing experiments have recently been used to track cancer cell growth in both skin and mammary tumours and have produced the first in vivo evidence of cancer stem cells as defined by tumour initiation $[43,44]$. In order to observe plasticity in vivo Zomer et al. [44] induced random expression of one of four colours in all tumour cells of the PyMT mouse model of spontaneous tumour formation. Using this system and by imaging at various stages during tumour progression it was possible to identify clonal populations by single colour expression which either expanded at a later stage of tumour formation or regressed, thereby implying loss or gain of the bCSC property of tumour initiation [44]. The regulation of this observed plasticity was not investigated in this study. To further expand our knowledge of plasticity and to investigate the underlying molecular mechanisms, it may be advantageous to develop functional plasticity assays; i.e. the elimination and reacquisition of a single functional stem-like attribute such as tumour initiation as mentioned above [43] or mammosphere formation as proposed by Piggott et al. [45]. 


\section{Plasticity within breast cancer stem-like cell populations}

Adding yet another layer of complexity to the issue of plasticity is the notion that there may exist within a tumour more than one distinct cancer stem-like state between which cells can transition, possibly depending on the influence of external cues. Evidence for multiple stem-like states can only come from studies which further purify the CSC component into putative subpopulations.

As mentioned previously, 2 putative subpopulations of bCSCs have been identified in breast tumour samples based on the two marker profiles of $\mathrm{ALDH}^{+}$and $\mathrm{CD} 44^{+} / \mathrm{CD} 24 / \mathrm{ESA}^{+}$[24]. In a recent review it was suggested that these two populations are present in distinct compartments of the normal mammary gland hierarchy and exist in EMT-like $\left(\mathrm{ALDH}^{+}\right)$, and MET-like $\mathrm{CD} 44^{+} / \mathrm{CD} 24^{-} / \mathrm{ESA}^{+}$states. This model suggests that interconversion between stem-like states can occur via an EMT like process, and also via MET. There is some evidence to suggest that interconversion is regulated by the expression of a number of microRNAs. It has been observed that the MET-like CSC population exhibits increased levels of mi93 which when overexpressed is capable of increasing this population further [31]. Consequently, inhibition of mi93 is able to induce EMT in isolated populations of ALDH ${ }^{+}$SUM159 cells. In contrast, upregulation of mi100 or 221 decreases the $\mathrm{ALDH}^{+}$ population and increases $\mathrm{CD} 44^{+} / \mathrm{CD} 24 / \mathrm{ESA}^{+}$cells. These data are reviewed in more detail in Liu et al. [31]. Whilst evidence for intrabCSC plasticity is still lacking, this group has demonstrated convincing support for this hypothesis which may explain a previous discrepancy in breast tumorigenesis; why EMT is known to increase metastasis but metastases are often more epithelial-like than the primary tumour $[31,46]$.

Further support for this theory comes from an investigation into the effect of hypoxia on tumour initiating cells. Hypoxic conditions were found to confer the property of invasiveness on a cell line enriched for tumour initiating cells (SK3rd), but which was previously stationary. This effect was mediated by membrane type 1 matrix metallo-proteinase (Mt1-MMP), the inhibition of which was found to reduce invasiveness under hypoxic conditions. Hypoxia was not able to affect the expression of Mt1-MMP, but instead altered its redistribution to the cell surface. Elevated levels of surface Mt1-MMP although associated with enhanced invasive ability correlated with a decrease in the bCSC marker CD44, and also disrupted mammosphere formation. However, hypoxia or elevated surface Mt1-MMP did not alter the tumorigenic capacity of the tumour initiating cells but the volume of lung metastases was increased. These data imply the existence of two distinct stem-like populations which can interconvert in response to oxygen levels in the tumour environment. In addition to regulation by hypoxia, EMT may also play a role in this plasticity. The non-invasive bCSCs in this model were found to be mesenchyme-like in comparison to the SKBR3 parental line. Twist1 was elevated in the SK3rd cells which in turn were found to be capable of regulating MT1MMP expression via miR10b and HoxD10. However as hypoxia is not able to regulate MT1-MMP expression, the relevance of EMT in this model remains to be firmly established. In addition, the authors did not report on the epithelial or mesenchymal status of the SK3rd cells under hypoxic conditions [47]. Despite this the findings of this study do correlate with the suggestion of Liu et al. [31] that these two stemlike populations may reside at different areas or niches of the tumour: the MET-like at the centre (hypoxic), and the EMT like at the invasive front (normoxic). Of most relevance is the finding that alterations to the oxygen level mimicking those occuring during metastasis can also alter the percentage of mammosphere-forming cells in the Sk3rd model thus strongly implicating hypoxia in the dynamic regulation of bCSC states [47] (Table 2).

\section{Conclusions}

At present, the resistance of stem-like cells to commonly prescribed anti-cancer drugs poses a major problem for the successful treatment of breast cancer patients. The ability of stem-like cells to survive treatments which otherwise shrink tumour growth suggests that they may be responsible for the disease recurrence which is observed in a large proportion of patients. This strongly suggests that a proportion of cells with tumorigenic capacity remain or have regenerated from among surviving tumour cells. Despite the complexity of CSCs, it is clear that stem-like properties contribute the most detrimental aspects of malignancy to a tumour. Understanding how stem-like attributes have become unregulated in tumours will undoubtedly lead to the identification of novel targets. However, the complexity of CSCs in terms of their heterogeneity and plasticity will make any one single drug unlikely to be efficient. An ideal strategy would be to target both the CSC and the non-stem populations in order to prevent the reacquisition of stem-like characteristics by plasticity. Research in this area taking these factors into consideration could lead to the development of therapeutic strategies with enormous potential for efficacy.

\section{References}

1. Reya T, Morrison SJ, Clarke MF, Weissman IL (2001) Stem cells, cancer, and cancer stem cells. Nature 414: 105-111.

2. Campbell LL, Polyak K (2007) Breast tumor heterogeneity: cancer stem cells or clonal evolution? Cell Cycle 6: 2332-2338.

3. Bonnet D, Dick JE (1997) Human acute myeloid leukemia is organized as a hierarchy that originates from a primitive hematopoietic cell. Nat Med 3: 730737.

4. Al-Hajj M, Wicha MS, Benito-Hernandez A, Morrison SJ, Clarke MF (2003) Prospective identification of tumorigenic breast cancer cells. Proc Natl Acad Sci USA 100: 3983-3988.

5. Singh SK, Hawkins C, Clarke ID, Squire JA, Bayani J, et al. (2004) Identification of human brain tumour initiating cells. Nature 432: 396-401.

6. O'Brien CA, Pollett A, Gallinger S, Dick JE (2007) A human colon cancer cell capable of initiating tumour growth in immunodeficient mice. Nature 445: 106 110.

7. Ricci-Vitiani L, Lombardi DG, Pilozzi E, Biffoni M, Todaro M, et al. (2007) Identification and expansion of human colon-cancer-initiating cells. Nature 445 111-115.

8. Hermann PC, Huber SL, Herrler T, Aicher A, Ellwart JW, et al. (2007) Distinct populations of cancer stem cells determine tumor growth and metastatic activity in human pancreatic cancer. Cell Stem Cell 1: 313-323.

9. Clevers H (2005) Stem cells, asymmetric division and cancer. Nat Genet 37 1027-1028.

10. Morrison SJ, Kimble J (2006) Asymmetric and symmetric stem-cell divisions in development and cancer. Nature 441: 1068-1074.

11. Greaves M (2010) Cancer stem cells: Back to Darwin? Semin Cancer Biol 20 65-70.

12. Cicalese A, Bonizzi G, Pasi CE, Faretta M, Ronzoni S, et al. (2009) The Tumor Suppressor p53 Regulates Polarity of Self-Renewing Divisions in Mammary Stem Cells. Cell 138: 1083-1095.

13. Chaffer CL, Brueckmann I, Scheel C, Kaestli AJ, Wiggins PA, et al. (2011) Normal and neoplastic nonstem cells can spontaneously convert to a stem-like state. Proc Natl Acad Sci USA 108: 7950-7955.

14. Gupta PB, Fillmore CM, Jiang G, Shapira SD, Tao K, et al. (2011) Stochastic state transitions give rise to phenotypic equilibrium in populations of cancer cells. Cell 146: 633-644.

15. Perou CM, Sørlie T, Eisen MB, van de Rijn M, Jeffrey SS, et al. (2000) Molecular portraits of human breast tumours. Nature 406: 747-752. 
Citation: French R, Clarkson R (2012) The Complex Nature of Breast Cancer Stem-Like Cells: Heterogeneity and Plasticity. J Stem Cell Res Ther S7:009. doi:10.4172/2157-7633.S7-009

Page 7 of 7

16. Prat A, Parker JS, Karginova O, Fan C, Livasy C, et al. (2010) Phenotypic and molecular characterization of the claudin-low intrinsic subtype of breast cancer. Breast Cancer Res 12: R68.

17. Hwang-Verslues WW, Kuo WH, Chang PH, Pan CC, Wang HH, et al. (2009) Multiple Lineages of Human Breast Cancer Stem/Progenitor Cells Identified by Profiling with Stem Cell Markers. PLoS One 4: e8377.

18. Honeth G, Bendahl PO, Ringnér M, Saal LH, Gruvberger-Saal SK, et al. (2008) The CD44+/CD24- phenotype is enriched in basal-like breast tumors. Breast Cancer Res 10: R53.

19. Meyer MJ, Fleming JM, Lin AF, Hussnain SA, Ginsburg E, et al. (2010) CD44posCD49fhiCD133/2hi Defines Xenograft-Initiating Cells in Estrogen Receptor-Negative Breast Cancer. Cancer Res 70: 4624-4633.

20. Stingl J, Eirew P, Ricketson I, Shackleton M, Vaillant F, et al. (2006) Purification and unique properties of mammary epithelial stem cells. Nat lett 439: 993-997.

21. Cariati M, Naderi A, Brown JP, Smalley MJ, Pinder SE, et al. (2008) Alpha-6 integrin is necessary for the tumourigenicity of a stem cell-like subpopulation within the MCF7 breast cancer cell line. Int J Cancer 122: 298-304.

22. Mizrak D, Brittan M, Alison M (2008) CD 133: molecule of the moment. J Patho 214: 3-9.

23. Wright $M H$, Calcagno AM, Salcido CD, Carlson MD, Ambudkar SV, et al. (2008) Brca1 breast tumors contain distinct CD44+/CD24- and CD133+ cells with cancer stem cell characteristics. Breast Cancer Res 10: R10.

24. Ginestier C, Hur MH, Charafe-Jauffret E, Monville F, Dutcher J, et al. (2007) ALDH1 is a marker of normal and malignant human mammary stem cells and a predictor of poor clinical outcome. Cell Stem Cell 1: 555-567.

25. Yoshida A, Rzhetsky A, Hsu LC, Chang C (1998) Human aldehyde dehydrogenase gene family. Eur J Biochem 251: 549-557.

26. Charafe-Jauffret E, Ginestier C, lovino F, Wicinski J, Cervera N, et al. (2009) Breast cancer cell lines contain functional cancer stem cells with metastatic capacity and a distinct molecular signature. Cancer Res 69: 1302-1313.

27. de Beça FF, Caetano P, Gerhard R, Alvarenga CA, Gomes M, et al. (2012) Cancer stem cells markers CD44, CD24 and ALDH1 in breast cancer special histological types. J Clin Pathol.

28. Vaillant F, Asselin-Labat ML, Shackleton M, Forrest NC, Lindeman GJ, et al (2008) The mammary progenitor marker CD61/B3 integrin identifies cancer stem cells in mouse models of mammary tumourigenesis. Cancer Res 68: 7711-7717.

29. Smalley M, Piggott L, Clarkson R (2012) Breast Cancer Stem cells: Obstacles to Therapy. Cancer Lett.

30. Biddle A, Liang X, Gammon L, Fazil B, Harper LJ, et al. (2011) Cancer Stem Cells in Squamous Cell Carcinoma Switch between Two Distinct Phenotypes That Are Preferentially Migratory or Proliferative Cancer Res 71: 5317-5326.

31. Liu S, Clouthier SG, Wicha MS (2012) Role of microRNAs in the Regulation of Breast Cancer Stem Cells. J Mammary Gland Biol Neoplasia 17: 15-21.

32. Zucchi I, Astigiano S, Bertalot G, Sanzone S, Cocola C, et al. (2008) Distinct populations of tumor-initiating cells derived from a tumor generated by rat mammary cancer stem cells. Proc Natl Acad Sci USA 105: 16940-16945.

33. Drasin DJ, Robin TP, Ford HL (2011) Breast cancer epithelial-to-mesenchymal transition: examining the functional consequences of plasticity. Breast Cancer Res 13: 226

34. Mani SA, Guo W, Liao MJ, Eaton EN, Ayyanan A, et al. (2008) The epithelialmesenchymal transition generates cells with properties of stem cells. Cell. 133 704-715.

35. Morel AP, Lièvre M, Thomas C, Hinkal G, Ansieau S, et al. (2008) Generation of breast cancer stem cells through epithelial-mesenchymal transition. PLoS One 3: e2888.

36. Blick T, Hugo H, Widodo E, Waltham M, Pinto C, et al. (2010) Epithelia Mesenchymal Transition Traits in Human Breast Cancer Cell Lines Paralle the CD44(hi/)CD24 (lo/-) Stem Cell Phenotype in Human Breast Cancer. J Mammary Gland Biol Neoplasia 15: 235-252.

37. Yang G, Quan Y, Wang W, Fu Q, Wu J, et al. (2012) Dynamic equilibrium between cancer stem cells and non-stem cancer cells in human SW620 and MCF-7 cancer cell populations. Br J Cancer 106: 1512-1519.

38. Bisaro B, Montani M, Konstantinidou G, Marchini C, Pietrella L, et al. (2012) p130Cas/Cyclooxygenase-2 axis in the control of mesenchymal plasticity of breast cancer cells. Breast Cancer Res 14: R137.

39. Alsarraj J, Walker RC, Webster JD, Geiger TR, Crawford NP, et al. (2011) Deletion of the Proline-Rich Region of the Murine Metastasis Susceptibility Gene Brd4 Promotes Epithelial-to-Mesenchymal Transition- and Stem CellLike Conversion. Cancer Res. 71: 3121-3131.

40. Kato M, Slack FJ (2008) microRNAs: Small molecules with big roles - C elegans to human cancer. Biol. Cell 100: 71-81.

41. Chang CJ, Chao CH, Xia W, Yang JY, Xiong Y, et al. (2011) p53 regulates epithelial-mesenchymal transition and stem cell properties through modulating miRNAs, Nat Cell Biol 13: 317-323.

42. Polytarchou C, lliopoulos D, Struhl K (2012) An integrated transcriptional regulatory circuit that reinforces the breast cancer stem cell state. Proc Nat Acad Sci USA 109: 14470-14475.

43. Driessens G, Beck B, Caauwe A, Simons BD, Blanpain C (2012) Defining the mode of tumour growth by clonal analysis. Nature 488: 527-530.

44. Zomer A, Inge Johanna Ellenbroek S, Ritsma L, Beerling E, Vrisekoop N, et al (2012) Intravital Imaging of Cancer Stem Cell Plasticity in Mammary Tumors Stem Cells.

45. Piggott L, Omidvar N, Martí Pérez S, Eberl M, Clarkson RW (2011) Suppression of apoptosis inhibitor c-FLIP selectively eliminates breast cancer stem cell activity in response to the anti-cancer agent, TRAIL. Breast Cancer Res 13 R88.

46. Shipitsin M, Campbell LL, Argani P, Weremowicz S, Bloushtain-Qimron N, et al. (2007) Molecular definition of breast tumor heterogeneity. Cancer Cell 11 259-273.

47. Li J, Zucker S, Pulkoski-Gross A, Kuscu C, Karaayvaz M, et al. (2012) Conversion of Stationary to Invasive Tumor Initiating Cells (TICs): Role of Hypoxia in Membrane Type 1-Matrix Metalloproteinase (MT1-MMP) Trafficking PLoS One 7: e38403.

48. Dontu G, Abdallah WM, Foley JM, Jackson KW, Clarke MF, et al. (2003) In vitro propagation and transcriptional profiling of human mammary stem/progenitor cells. Genes Dev 17: 1253-1270.
This article was originally published in a special issue, Cancer Stem Cells handled by Editor(s). Fazlul Hoque Sarkar, Wayne State University, USA; Asfar Sohail Azmi, Wayne State University, USA 